\title{
A NUMERICAL SOLUTION TO THE FLOW BETWEEN ECCENTRIC ROTATING CYLINDERS WITH A SLOTTED SLEEVE
}

\author{
L. D. HIRD ${ }^{1}$, P. F. SIEW ${ }^{1}$ and S. WANG ${ }^{1}$
}

(Received 17 March 1997; revised 22 April 1998)

\begin{abstract}
The flow between two eccentric rotating cylinders with a slotted sleeve placed around the inner cylinder is determined numerically using an exponentially fitted finite-volume method. The flow field is determined for various Reynolds numbers, eccentricities and rotational speeds for the cases when the cylinders rotate in the same sense and rotate in opposite senses. The flow field developed when both cylinders rotate in the same sense is characterised, for sufficiently large eccentricity and rotational rate, by two counter-rotating eddies. Only one eddy is observed when the cylinders rotate in opposite senses. The presence of these eddies restricts the flow through the slotted sleeve in the former case but encourages through flow in the latter. For both cases, the eccentricity affects the location of the eddies, while changing the relative rotational rate only affects the eddy location for the case when the cylinders rotate in opposite directions. The change in Reynolds number has little effect on the flow field for the problems considered here. The vorticity generated by the slotted sleeve is convected into the main body of the flow field. No inviscid core within the main body of the flow field is observed for the range of Reynolds number considered.
\end{abstract}

\section{Introduction}

This paper examines the numerical solution of the incompressible Navier-Stokes equations governing the flow between two eccentric rotating cylinders with a slotted sleeve placed around the inner cylinder. The setup is a two-dimensional model of the modified viscometer used to estimate the rheological properties of settling slurries, and assumes that end effects in the viscometer are unimportant. This apparatus was first considered by Overend et al. [20]. Very little is known about the flow field of the slurry, and, in practice, surface chemistry effects need to be considered. In order to see how the slotted sleeve affects the flow field, Hird and Siew [13] first considered the case of a Newtonian fluid in the small Reynolds number limit. The sleeve is assumed

'Curtin University of Technology, GPO Box U1987, Perth, Western Australia 6845

(C) Australian Mathematical Society 1999, Serial-fee code 0334-2700/99 
to have numerous small slots so that a good approximation is to replace it with a rigid permeable membrane on which a no-slip condition is imposed. The low Reynolds number limit allows the solution of the flow field to be investigated analytically.

In practice, the slots in the modified viscometer are large enough to encourage entrainment of the slurry onto the rotor. Also, the average density of the slurry may vary according to its composition. It is, therefore, worthwhile to look at the flow field when the slots are large, and, to simplify the model, we restrict ourselves to a homogeneous fluid with Reynolds number covering a reasonable range (50-1000, say). High Reynolds number flows are characterised by the presence of boundary layers along no-slip surfaces, and within these layers the velocity gradient normal to the surface is large. Methods based on centred-difference approximations to the advective term often result in solutions exhibiting oscillatory behaviour close to these layers. Upwind schemes are often used to suppress these oscillations; however, firstorder schemes are known to give inaccurate results [3]. While higher-order schemes may overcome this, they are computationally more complicated (see for example [26]). Here we use the exponentially fitted finite-volume method based on Delaunay triangulations and Voronoi tessellations used by Miller and Wang $[18,19]$. This method reduces to the centred and upwind difference schemes, respectively for low and high Reynolds numbers, and is shown to be an improvement over these methods for triangulations based on standard finite-difference meshes [19].

This paper is organised as follows. Firstly, the stream function-vorticity formulation is used to define the governing equations and the boundary conditions defining the problem are stated. Secondly, the finite-volume method defined in [18] is used to generate a system of coupled algebraic equations. Thirdly, the techniques used to estimate the unknown Dirichlet conditions are given. Finally, the iterative algorithm used to solve the system of algebraic equations is outlined. The effects on the flow field due to eccentricity, Reynolds number, and the speed and direction of rotation of the cylinders are discussed. The stability of the flow between eccentric rotating cylinders has been studied by various authors (see for example [8]). The existence of the slotted sleeve appears to have a stabilising effect on the flow field.

\section{Governing differential equations and boundary conditions}

The flow region consists of an inner cylinder (called the rotor) of radius $c$ rotating uniformly with angular velocity $\omega_{1}$ surrounded by a stationary sleeve and having radius $a$. This assembly is then placed with its centre a distance $a \epsilon$ from the centre of the outer cylinder or bowl. The bowl, having radius $b$, rotates uniformly with angular velocity $\omega_{2}$ (see Figure 1 ). Let $\Omega$ represent the region of the $x y$-plane, between the two cylinders, with origin at the centre of the rotor, and let $\partial \Omega_{1}$ and $\partial \Omega_{2}$ denote the 
rotor and bowl, respectively. Also let $\partial \Omega_{3}^{q}(q=1, \ldots, s)$ denote the surface elements of the sleeve consisting of $s$ surface elements. For notational ease, $\partial \Omega_{3}$ is used when it is not necessary to identify $\partial \Omega_{3}^{q}$ individually.

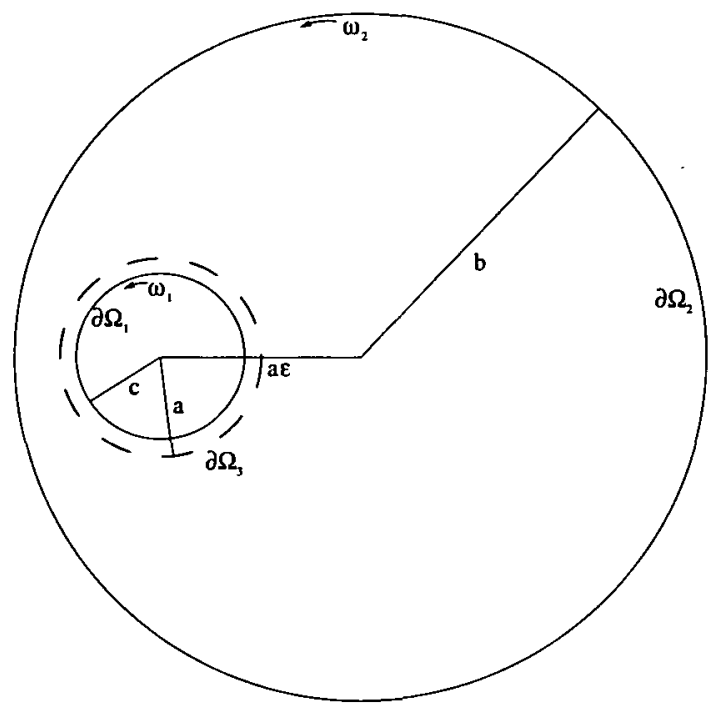

FIGURE 1. Layout.

At a point $\mathbf{x}\left(=\left(x_{i}, y_{i}\right)\right)$ in $\Omega$ and at a given time $t \in[0, \mathrm{~T}]$, the Navier-Stokes equations governing the laminar flow of an incompressible homogeneous Newtonian fluid (that is, having constant kinematic viscosity, $v$ ) are given by

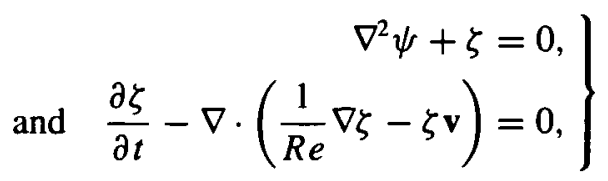

where $\psi$ represents the stream function, $\zeta$ represents the component of vorticity normal to $\Omega$, and $\mathbf{v}$ is the velocity vector. The above equations are written in dimensionless form with the Reynolds number given by $R e=\frac{a b \omega_{2}}{v}$ where $a$ and $b \omega_{2}$ represent, respectively, the characteristic length and velocity scales. The no-slip conditions on the solid surfaces $\partial \Omega_{1}, \partial \Omega_{2}$ and $\partial \Omega_{3}$ yield the boundary conditions

$$
\text { and } \left.\begin{array}{rl}
\frac{\partial \psi}{\partial \mathbf{n}}=\bar{U} & \forall \mathbf{x} \in \partial \Omega_{1} \text { and } t \in[0, \mathrm{~T}], \\
\frac{\partial \psi}{\partial \mathbf{n}}=-1 & \forall \mathbf{x} \in \partial \Omega_{2} \text { and } t \in[0, \mathrm{~T}], \\
=0 & \forall \mathbf{x} \in \partial \Omega_{3} \text { and } t \in[0, \mathrm{~T}],
\end{array}\right\}
$$


where $\mathbf{n}$ represents the unit outward normal vector and $\bar{U}=\frac{c \omega_{1}}{b \omega_{2}}$ denotes the dimensionless azimuthal velocity of the rotor. Along each of the boundaries, the stream function is represented by

$$
\psi=0 \forall \mathbf{x} \in \partial \Omega_{1}, \quad \psi=K_{2} \forall \mathbf{x} \in \partial \Omega_{2}, \quad \text { and } \quad \psi=K_{3}^{q} \forall \mathbf{x} \in \partial \Omega_{3}^{q}, q=1, \ldots, s .
$$

These correspond to no flow through the solid boundaries. As the normal derivatives in (2.2) determine the stream function up to an additive constant, $K_{2}$ and $K_{3}^{q}$ must be found as part of the solution process; they will be approximated from the nodal values local to $\partial \Omega_{2}$ and $\partial \Omega_{3}$. Also, the above Neumann conditions (2.2), together with (2.1), will be used to prescribe $\zeta$ along the no-slip boundaries.

Our principal aim is to solve stationary problems whose solutions are regarded as the long-term solution of the transient problem given above.

\section{A finite volume formulation}

A complete discussion of the finite-volume method used here can be found in $[18,19]$. To discretise the above governing equations we introduce the Delaunay triangulation, $T_{h}$, with mesh parameter $h$, and its dual the Voronoi (Dirichlet) tessellation, $D_{h}$, of the domain $\Omega$ (see Figure 2). Each vertex, $\mathbf{x}_{i}$, of $T_{h}$ is associated with a convex set, $d_{i}$, whose boundary, $\partial d_{i}$, is a Voronoi polygon with vertices being the circumcentres of all triangles with common vertex $\mathbf{x}_{i}$. Following the treatment in [18] we let $l_{i j}$ denote the edge of $\partial d_{i}$ normal to $e_{i j}$ which joins $\mathbf{x}_{i}$ and $\mathbf{x}_{j}$.

Integrating over $d_{i}$, the vorticity equation becomes

$$
\zeta_{i}^{k}+\sum_{j \in l_{i}} \frac{\Delta t_{k} \sigma_{i j}}{\left|d_{i}\right|}\left[B\left(-R e \bar{v}_{i j}^{k}\left|e_{i j}\right|\right) \zeta_{i}^{k}-B\left(R e \bar{v}_{i j}^{k}\left|e_{i j}\right|\right) \zeta_{j}^{k}\right]=\zeta_{i}^{k-1}, \quad i=1, \ldots, N_{v}
$$

where $\sigma_{i j}=\frac{\left|l_{i j}\right|}{R e\left|e_{i j}\right|},\left|d_{i}\right|$ is the area of $d_{i}, N_{v}$ is the number of nodes not on a boundary, the superscript $k$ refers to the $k$ th time step, and $B(x)=x /\left(e^{x}-1\right)$ for $x \neq 0$, with $B(0)=1$, is the Bernoulli function. Also $\bar{v}_{i j}=\left(\psi_{i j ; 2}-\psi_{i j ; 1}\right) /\left|l_{i j}\right|$, where $\psi_{i j ; 2}$ and $\psi_{i j ; 1}$ represent the nodal values of $\psi$ at the end-points of $l_{i j}$, estimated from the nodal values of the vertices of the triangles with circumcentres at $\mathbf{x}_{i j ; 2}$ and $\mathbf{x}_{i j ; 1}$. A backward Euler scheme, which is known to be unconditionally stable, has been used, and $\Delta t_{k}=t_{k}-t_{k-1}$.

Written in matrix notation, we have

$$
\left(I+C^{k}\right) Z^{k}+W^{k}=Z^{k-1},
$$




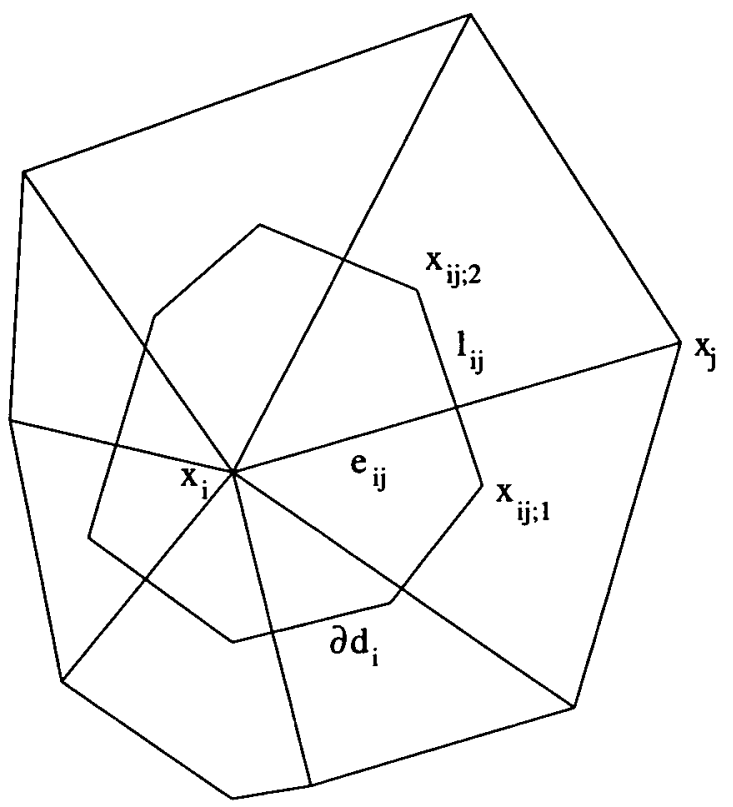

FIGURE 2. The Voronoi tessellation and Delaunay mesh around a mesh node.

where $I$ is the identity matrix,

$$
\begin{aligned}
c_{i i}^{k} & =\Delta t_{k} \sum_{j \in I_{i}} \frac{\sigma_{i j}}{\left|d_{i}\right|} B\left(\frac{\psi_{i j: 1}^{k}-\psi_{i j ; 2}^{k}}{\sigma_{i j}}\right), \\
c_{i j}^{k} & =-\Delta t_{k} \frac{\sigma_{i j}}{\left|d_{i}\right|} B\left(\frac{\psi_{i j ; 2}^{k}-\psi_{i j ; 1}^{k}}{\sigma_{i j}}\right) \quad \text { if } \mathbf{x}_{j} \notin \partial \Omega_{1} \cup \partial \Omega_{2} \cup \partial \Omega_{3}, \\
w_{i}^{k} & = \begin{cases}-\sum_{j \in I_{i}} \Delta t_{k} \frac{\sigma_{i j}}{\left|d_{i}\right|} B\left(\frac{\psi_{i j ; 2}^{k}-\psi_{i j ; 1}^{k}}{\sigma_{i j}}\right) \zeta_{1}^{j} & \text { if } \mathbf{x}_{j} \in \partial \Omega_{1} \\
-\sum_{j \in I_{i}} \Delta t_{k} \frac{\sigma_{i j}}{\left|d_{i}\right|} B\left(\frac{\psi_{i j: 2}^{k}-\psi_{i j ; 1}^{k}}{\sigma_{i j}}\right) \zeta_{2}^{j} & \text { if } \mathbf{x}_{j} \in \partial \Omega_{2} \\
-\sum_{j \in I_{i}} \Delta t_{k} \frac{\sigma_{i j}}{\left|d_{i}\right|} B\left(\frac{\psi_{i j ; 2}^{k}-\psi_{i j ; 1}^{k}}{\sigma_{i j}}\right) \zeta_{3, q}^{j} & \text { if } \mathbf{x}_{j} \in \partial \Omega_{3}^{q}, \\
0 & \text { if } \mathbf{x}_{j} \notin \partial \Omega_{1} \cup \partial \Omega_{2} \cup \partial \Omega_{3}\end{cases}
\end{aligned}
$$

and

$$
z_{i}^{k}=\zeta_{i}^{k}
$$


Here $\zeta_{1}^{j}, \zeta_{2}^{j}$, and $\zeta_{3, q}^{j}$ represent the vorticity at node $\mathbf{x}_{j}$ along the no-slip boundaries, and can be estimated at each time step through equations (4.4), (4.5), and (4.6) as shown in Section 4. The Poisson equation in (2.1) can be discretised in the same manner leading to

$$
\sum_{j \in I_{i}} \frac{\left|l_{i j}\right|}{\left|e_{i j}\right|\left|d_{i}\right|}\left(\psi_{i}^{k}-\psi_{j}^{k}\right)=\zeta_{i}^{k}, \quad i=1, \ldots, N_{v}
$$

or equivalently

$$
A \Psi^{k}+B^{k}=Z^{k}
$$

where

$$
\begin{aligned}
& a_{i i}=\sum_{j \in I_{i}} \frac{\left|l_{i j}\right|}{\left|e_{i j}\right|\left|d_{i}\right|}, \\
& a_{i j}=-\frac{\left|l_{i j}\right|}{\left|e_{i j}\right|\left|d_{i}\right|} \quad \text { if } \mathbf{x}_{j} \notin \partial \Omega_{1} \cup \partial \Omega_{2} \cup \partial \Omega_{3}, i \neq j
\end{aligned}
$$

and

$$
b_{i}^{k}= \begin{cases}-\sum_{j \in I_{i}} \frac{\left|l_{i j}\right|}{\left|e_{i j}\right|\left|d_{i}\right|} K_{2} & \text { if } \mathbf{x}_{j} \in \partial \Omega_{2} \\ -\sum_{j \in I_{i}} \frac{\left|l_{i j}\right|}{\left|e_{i j}\right|\left|d_{i}\right|} K_{3}^{q} & \text { if } \mathbf{x}_{j} \in \partial \Omega_{3}^{q}, q=1, \ldots, s \\ 0 & \text { if } \mathbf{x}_{j} \notin \partial \Omega_{2} \cup \partial \Omega_{3} .\end{cases}
$$

\section{Estimating the Dirichlet boundary conditions}

4.1. Estimates for $K_{2}$ and $K_{3}^{q}$ The stream function value along $\partial \Omega_{1}$ is set to zero, but $K_{2}$ and $K_{3}^{q}$ must be determined a posteriori. Here we discuss the estimates for $K_{2}$ and $K_{3}^{q}$.

Given a Delaunay triangulation, $K_{2}$ and $K_{3}^{q}$ are estimated from the interior nodal values of the stream function as follows. The Dirichlet boundary condition $K_{3}^{q}$ along each surface element of $\partial \Omega_{3}^{q}, q=1, \ldots, s$, is estimated using the mean value of the $2 n$ nodes surrounding each surface element. Hence

$$
K_{3}^{q} \approx \frac{1}{2 n} \sum_{j=1}^{n}\left(\psi_{j, q+1}+\psi_{j, q-1}\right)=\bar{K}_{3}^{q}, \quad q=1,2, \ldots, s,
$$

where $\psi_{j, q+1}$ and $\psi_{j, q-1}$ represent the $j^{\text {th }}$ nodal value above and below surface element $\partial \Omega_{3}^{q}$ respectively. 
Consider a Delaunay triangle with vertices $x_{1}, x_{2}$, and $x_{3}$, and only one vertex lying on $\partial \Omega_{2}$ ( $x_{3}$ say) (see Figure 3). To approximate $\psi$ on $\partial \Omega_{2}$ we use a linear approximation at each such boundary node, $\psi_{j, b_{2}}, j=1, \ldots, \alpha$, given by

$$
\psi_{j, b_{2}}=\psi_{j, b_{2}-1}+\left.\delta_{j} \frac{\partial \psi}{\partial \mathbf{n}}\right|_{\partial \Omega_{2}}+O\left(\delta_{j}^{2}\right), \quad j=1, \ldots, \alpha, \text { where }
$$

$\psi_{j, b_{2}-1}$ is an adjacent point at a distance of $\delta_{j}$ from the boundary in the normal direction and is given by

$$
\psi_{j, b_{2}-1} \approx S\left(\mathbf{x}^{*}\right) \psi\left(\mathbf{x}_{2}\right)+\left(1-S\left(\mathbf{x}^{*}\right)\right) \psi\left(\mathbf{x}_{1}\right)
$$

where $S\left(\mathbf{x}^{*}\right)=\frac{\left\|\mathbf{x}_{1}-\mathbf{x}^{*}\right\|_{2}}{\left\|\mathbf{x}_{1}-\mathbf{x}_{2}\right\|_{2}}$ and $\|\cdot\|_{2}$ represents the Euclidean norm. $K_{2}$ is estimated from the arithmetic mean of $\psi_{j, b_{2}}$, viz.,

$$
K_{2} \approx \frac{1}{\alpha} \sum_{j=1}^{\alpha}\left(\psi_{j, b_{2}-1}-\delta_{j}\right)=\bar{K}_{2}
$$

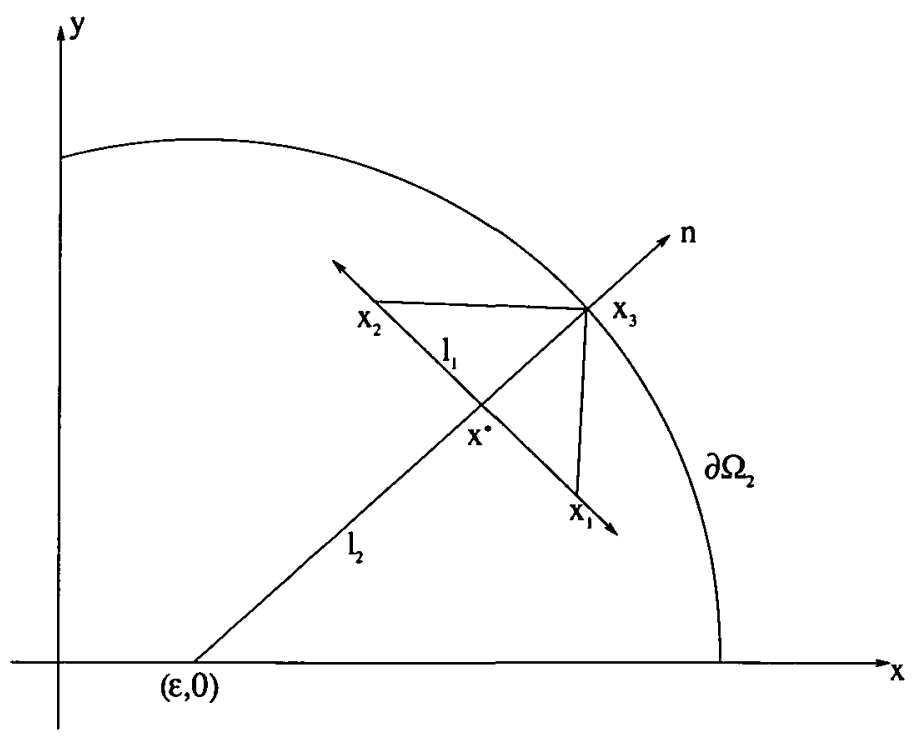

FIGURE 3. Interpolating to get $\psi$ on $\partial \Omega_{2}$.

4.2. Approximating $\zeta$ on the no-slip boundaries Although the equations given in (2.1) are coupled and have been solved as such (see $[4,9,10]$ ), many workers have had success decoupling these by approximating the vorticity along no-slip surfaces from 
the discretised Poisson equation (see $[1,6,15,18,23]$ ). We follow the approach as described in [18]. Over each boundary tessellation, integrating the Poisson equation in (2.1), and using the Neumann and Dirichlet conditions given in (2.2) and (2.3), leads to the vorticity boundary estimates at node $x_{b}$ on boundary $\partial \Omega_{i}(i=1,2,3)$, namely $\zeta_{i}^{b}$ given by

$$
\begin{aligned}
& \zeta_{1}^{b} \approx-\frac{1}{\left|d_{b}\right|}\left[\sum_{j \in I_{b}, x_{j} \notin \partial \Omega_{1}} \psi_{j}^{k} \frac{\left|l_{b j}\right|}{\left|e_{b j}\right|}+\int_{\partial d_{b} \cap \partial \Omega_{1}} \bar{U} d s\right], \\
& \zeta_{2}^{b} \approx-\frac{1}{\left|d_{b}\right|}\left[\sum_{j \in I_{b}, x_{j} \notin \partial \Omega_{2}}\left(\psi_{j}^{k}-K_{2}\right) \frac{\left|l_{b j}\right|}{\left|e_{b j}\right|}-\int_{\partial d_{b} \cap \partial \Omega_{2}} d s\right],
\end{aligned}
$$

and

$$
\zeta_{3, q}^{b} \approx-\frac{1}{\left|d_{b}\right|}\left[\sum_{j \in I_{b}, \boldsymbol{x}_{j} \notin \partial \Omega_{3}^{q}}\left(\psi_{j}^{k}-K_{3}^{q}\right) \frac{\left|l_{b j}\right|}{\left|e_{b j}\right|}\right], q=1, \ldots, s,
$$

where exact integration is performed on the integrals along $\partial \Omega_{1}$ and $\partial \Omega_{2}$, and where $K_{2}$ and $K_{3}^{q}$ are estimated at each time step using (4.3) and (4.1).

\section{An iterative procedure}

One disadvantage of using the stream function-vorticity formulation for multiconnected domains is that the stream function may be completely specified on only one boundary. The additive constants at the remaining boundaries must be determined as part of the solution process. The constants are often determined by imposing the condition that the pressure field is single-valued within the domain. This condition introduces an extra equation involving the stream function and vorticity from which the constants can be determined, see for example [11]. Here, we use a Gauss-Seidel iterative method to solve the coupled equations (3.1) and (3.6) with the boundary conditions (4.1), and (4.3) to (4.6) (see [12] for an outline of the scheme). In order to incorporate (4.1) and (4.3) into the iterative scheme, the discretised Poisson equation together with (4.1) and (4.3) are solved iteratively until self-consistent stream function and Dirichlet boundary values are obtained. The Dirichlet boundary values are smoothed between iterations to aid in the rate of convergence. Intuitively, this additional iterative scheme should not significantly affect the stability of the GaussSeidel scheme as we have smoothed the boundary values of $\psi$ before the vorticity vector is updated. However, the rate of convergence is expected to be affected, as the accuracy of $\bar{K}_{2}$ and $\bar{K}_{3}^{q}$ is dependent on how far the current vorticity field is from the steady-state solution. 
The solution to the Couette flow between the rotor and bowl is used to generate the initial estimates for the vorticity field and the stream function on $\partial \Omega_{2}$ and $\partial \Omega_{3}$. Three iterative schemes, referred to as the outer, middle, and inner iterations respectively, are then used to obtain steady-state solution fields. The outer iteration generates the sequence of solution fields converged at each time step, eventually reaching the steady-state solution. The middle iteration solves the discretised Poisson and vorticity transport equations through the use of the estimates for the vorticity along the solid boundaries given in (4.4), (4.5), and (4.6). The boundary estimates for $\zeta$ are updated using a weighted average, and convergence is reached when the difference between two successive estimates (in the maximum norm) is smaller than a given tolerance. When convergence is reached, the solution fields for a given time step are known. The inner iteration solves the discretised Poisson equation and updates $\bar{K}_{2}$ and $\bar{K}_{3}^{q}$ until self-consistent solutions are obtained. The converged results represent an intermediate set of values of the middle iteration. If superscript $k$ is used to represent the $k^{\text {th }}$ time step, superscript $j$ is used to represent the $j^{\text {th }}$ iterative step in the middle iteration, and superscript $i$ is used to represent the $i^{\text {th }}$ step of the inner iteration, then the modified Gauss-Seidel iterative scheme is as follows.

1. Choose tolerances $\epsilon_{0}, \epsilon_{1}$, and $\epsilon_{2}$, and smoothing parameters $\delta_{1}, \delta_{2} \in[0,1]$. Use the Couette flow to get the initial $Z^{j, k}, \bar{K}_{2}^{i, j, k}$, and $\bar{K}_{3}^{i, j, k}$ distributions and put $i, j, k=0$.

2. Solve (3.6) for $\Psi^{i+1, j+1, k+1}$, that is,

$$
A \Psi^{i+1, j+1, k+1}+B^{i+1, j+1, k+1}=Z^{j, k} .
$$

3. Update $\bar{K}_{2}^{i+1, j+1, k+1}$ and $\bar{K}_{3}^{i+1, j+1, k+1}$ using (4.3) and (4.1) and the smoothing parameter $\delta_{2}$, that is,

$$
\begin{aligned}
& \bar{K}_{2}^{i+1, j+1, k+1}:=\left(1-\delta_{2}\right) \bar{K}_{2}^{i+1, j+1, k+1}+\delta_{2} \bar{K}_{2}^{i, j+1, k+1}, \\
& \bar{K}_{3}^{i+1, j+1, k+1}:=\left(1-\delta_{2}\right) \bar{K}_{3}^{i+1, j+1, k+1}+\delta_{2} \bar{K}_{3}^{i, j+1, k+1} .
\end{aligned}
$$

4. Put $i=i+1$ and repeat steps $2-3$ until the convergence criteria

$$
\left|\bar{K}_{2}^{i+1, j+1, k}-\bar{K}_{2}^{i, j+1, k}\right|<\epsilon_{0} \text { and }\left\|\bar{K}_{3}^{i+1, j+1, k}-\bar{K}_{3}^{i, j+1, k}\right\|_{\infty}<\epsilon_{0}
$$

are met.

5. Put $\Psi^{j+1, k+1}=\Psi^{i+1, j+1, k+1}, \bar{K}_{2}^{j+1, k+1}=\bar{K}_{2}^{i+1, j+1, k+1}$, and $\bar{K}_{3}^{j+1, k+1}=\bar{K}_{3}^{i+1, j+1, k+1}$, and approximate vorticity, $Z_{b}^{j+1, k+1}$, at the no-slip surfaces, using (4.4), (4.5), and (4.6). Modify the values, using the smoothing parameter $\delta_{1}$, according to

$$
Z_{b}^{j+1, k+1}:=\left(1-\delta_{1}\right) Z_{b}^{j+1, k+1}+\delta_{1} Z_{b}^{j, k+1} .
$$


6. Solve (3.1) for $Z^{j+1, k+1}$, that is,

$$
B\left(I+C^{j+1, k+1}\right) Z^{j+1, k+1}+W^{j+1, k+1}=Z^{k} .
$$

7. Put $j=j+1, i=0$, and repeat steps $2-6$ until the convergence criterion $\left\|Z^{j+1, k+1}-Z^{j, k+1}\right\|_{\infty}<\epsilon_{1}$ is met.

8. Use $Z^{j+1, k+1}, Z_{b}^{j+1, k+1}, \Psi^{j+1, k+1}, \bar{K}_{2}^{j+1, k+1}$, and $\bar{K}_{3}^{j+1, k+1}$ to define the nodal values of vorticity and stream function $\left(\tilde{Z}^{k+1}, \tilde{\Psi}^{k+1}\right)$, at time step $k+1$.

9. Put $k=k+1, i, j=0$, and repeat steps $2-8$ until

$$
\left\|\tilde{Z}^{k+1}-\tilde{Z}^{k}\right\|_{\infty}<\epsilon_{2} .
$$

Here $\|\cdot\|_{\infty}$ represents the maximum norm. If the convergence criterion (5.1) is satisfied after $k$ steps, the program is continued for another $k$ time steps before the program terminates. This technique is used to avoid instances when spurious steady-state solutions are reached quickly, as are known to occur when the mesh is not fine enough. A non-dimensional time step of 0.1 is used for the problems considered in this paper. Since the time scale is $a /\left(b \omega_{2}\right)$, the equivalent real time step can be very small. For example, a rotational rate of $60 \mathrm{rpm}$ gives the real time step as less than 0.005 seconds. The solution fields obtained when convergence is first met are compared with the solution fields after the extended period of time before contour plots are produced. The Gauss-Seidel approach reduces the number of iterations as the nodal values reach the steady-state solution; further, it is not neccessary at the beginning of the procedure to solve the coupled system exactly at each time step as this is equivalent to restarting the iterative procedure at the next time step with an initial guess closer to the steady-state solution.

We note that the use of the smoothing parameters $\delta_{1}$ and $\delta_{2}$ as an aid to obtain steady-state solutions to coupled systems is not widely accepted. According to Gupta and Manohar [12] their use is essential for the convergence of the iterative scheme; however, Crochet, Davies and Walters [7, pages 138-139] stated that the use of a smoothing parameter is questionable, but, rather, the choice of the tolerances and initial guess influence the rate of convergence more than the need to use a smoothing parameter. We found the use of smoothing parameters a necessary feature of the iterative scheme here. Values of 0.95 and 0.8 are used for $\delta_{1}$ and $\delta_{2}$ respectively. The convergence rate is highly dependent on these parameters, in particular on the choice of $\delta_{1}$. It was impossible to converge the solution without some smoothing and indeed no convergence was obtained when $\delta_{1}$ was less than about 0.6. Also, a tolerance of $10^{-4}$ for $\epsilon_{0}, \epsilon_{1}$, and $\epsilon_{2}$ is used.

The sparsity of the matrices created makes the use of iterative schemes advantageous. The system given in (3.1) may be solved using the ICCG scheme [17], while (3.6) is solved using the conjugate-gradients-squared method (CGS) [22]. Further, incomplete $L U$ preconditioning of the matrices $\mathrm{A}$ is used to accelerate the convergence 
of the scheme. This combination of iterative scheme and preconditioning has been shown to be an efficient method of solving the vorticity transport equation [14,22].

Given the intended application of the apparatus, the torque exerted on the rotor about its centre is estimated at the end of each time step. If $T$ represents the dimensionless torque exerted on the rotor $\left(r=c / a=c^{*}\right)$ then, using polar coordinates, $T$ is given by

$$
T=\left.\int_{0}^{2 \pi} \tau_{r \theta}\right|_{r=c^{*}} d \theta
$$

where the dimensionless shear stress, $\tau_{r \theta}$, along the rotor is given by

$$
\left.\tau_{r \theta}\right|_{r=c^{*}}=\left.\left(\zeta-\frac{2 v_{\theta}}{r}\right)\right|_{r=c^{*}} .
$$

Hence,

$$
T=\left.\int_{0}^{2 \pi} \zeta\right|_{r=c^{*}} d \theta-\frac{4 \pi \bar{U}}{c^{*}}
$$

The trapezoidal rule is then used to evaluate $T$ from the vorticity data along the rotor.

\section{Numerical results and discussion}

To implement the numerical scheme used in this paper, the necessary codes were written in FORTRAN 77 using double precision in all computations, and run on an SGI INDY workstation.

When considering the two-dimensional model, the dimensions used represent the dimensions of the rheological apparatus. In MKS units, $a=0.024 \mathrm{~m}, b=0.093 \mathrm{~m}$, and $c=0.019 \mathrm{~m}$. Furthermore, 10 surface elements $(s=10)$ are used to represent the surface of the slotted sleeve. The sleeve used in practice is designed to facilitate the entrainment of the slurry onto the rotor. Typically, only $1 / 3$ of the sleeve is solid so a ratio of $1: 2$ for the surface to slot length is used in the current model.

The flow domain, $\Omega$, is initially partitioned into three disjoint regions; these regions cover the domain around the rotor (region $R 1$ ), the domain around the slotted sleeve (region $R 2$ ), and the domain of $\Omega$ a small distance from the slotted sleeve to the outer boundary (region $R 3$ ). Mesh nodes within $R 1$ and $R 3$ are generated through polar coordinates by incrementing the polar angle and radial distance through a prescribed number of intervals. Mesh nodes in $R 2$ are structured so that 5 nodes (equally spaced) lie along each surface element and 7 nodes (equally spaced) are placed along each slot. This sequence of nodes is repeated 3 times, a small distance both above and below the 
sleeve giving a total of 840 nodes concentrated along 7 circles a small distance around the slotted sleeve. For flows at large Reynolds numbers boundary layers on both sides of each surface element are expected to exist, therefore such a dense distribution of nodes around the sleeve is required to capture the behaviour in the shear layers around the slotted sleeve. The location of the nodes in $R 2$ is important in estimating $K_{3}^{q}$, as well as modelling the flow through the sleeve; therefore, the nodes in this mesh are not moved. However, the placement of the nodes in $R 1$ and $R 3$ are not important in estimating $K_{2}$ and $K_{3}^{q}$; they are simply used to cover their respective domains. The nodes in $\Omega$ are triangulated and the resulting mesh is smoothed by moving each interior node to the average of its neighbouring nodes. Smoothing the mesh so as to maximise the minimum angle and minimise the maximum angle is seen to yield more accurate results [18]. This mesh can be refined, if necessary, by adding a midpoint for each edge. Figure 4 shows a typical initial smoothed mesh and a subsequent refined one.

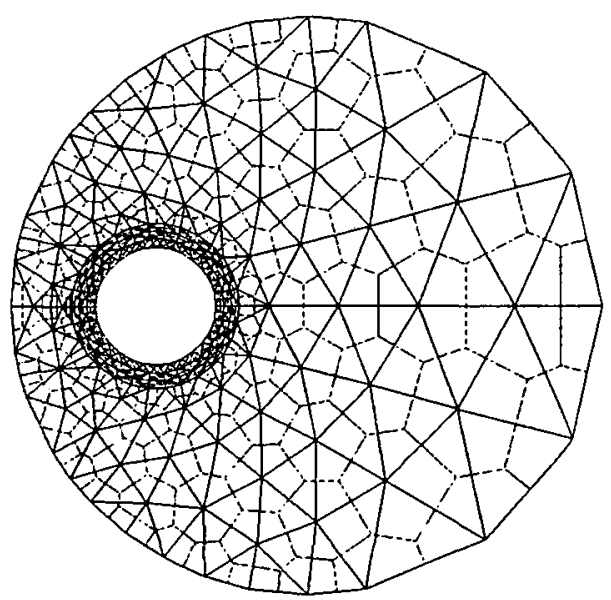

(a) Initial smoothed mesh

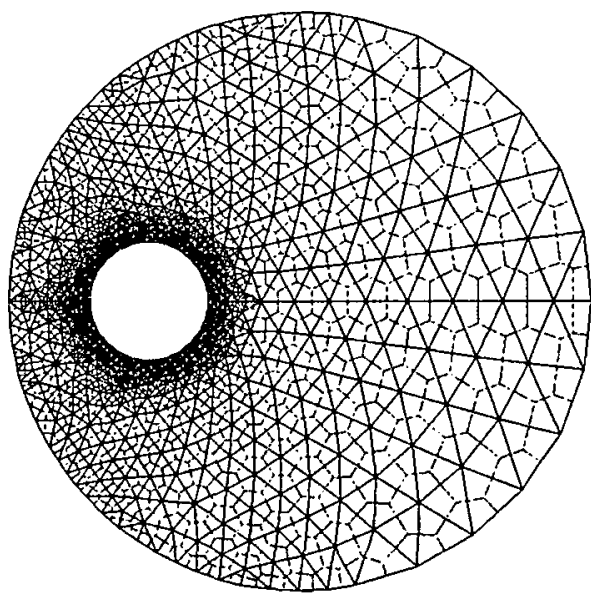

(b) Refined mesh

FIGURE 4. Mesh smoothing and refinement.

To study the flow around the slotted sleeve, we consider cases when the cylinders rotate in the same sense (case 1) and in opposite senses (case 2). For case 1, 0.5 and 0.7 are used for the eccentricity, $\bar{\epsilon}$, and the rotational speed of the rotor, $\bar{U}$, is varied up to 10.0. For case 2, eccentricities of $0.2,0.5$, and 0.7 , together with rotational speeds of the rotor up to -20.0 , are used. For both cases, the Reynolds number, $R e$, varies from 50 to 1000 .

From here on, the parameter set $\{R e, \bar{\epsilon}, \bar{U}\}$ is used to define a given problem, where the eccentricity is defined as the ratio of displacement centres to the mean radial clearance between the sleeve and outer cylinder, that is, $\bar{\epsilon}=a \epsilon /(b-a)$. The 
contour plots are generated using MATLAB [16].

To ensure that the numerical scheme will lead to mesh independent convergence, a problem is solved with successively refined meshes until the results, for the maximum (nodal) stream function, $|\psi|$, and the Dirichlet conditions along the slotted sleeve and the outer cylinder, are within $5 \%$ of one another for two consecutive meshes. In most cases, much better agreement is obtained. Table 1 shows the results from the problem $\{500.0,0.2,5.0\}$ using meshes with $1311,2503,5000$, and 9703 nodes respectively. In this case, the results for the last two meshes are within less than $1 \%$ of one another.

TABLE 1. Results for $(500.0,0.2,5.0\}$ using successively finer meshes.

\begin{tabular}{crrrrrrr}
\hline mesh & $\psi \mid \partial \Omega_{2}$ & $\max |\psi|$ & $\psi \mid \partial \Omega_{3}^{1}$ & $\psi \mid \partial \Omega_{3}^{2}$ & $\psi \mid \partial \Omega_{3}^{3}$ & $\psi \mid \partial \Omega_{3}^{4}$ & $\psi \mid \partial \Omega_{3}^{5}$ \\
\hline 1 & -4.3964 & 4.3964 & -0.6560 & -0.6649 & -0.6807 & -0.7013 & -0.7199 \\
2 & -4.4519 & 4.4519 & -0.6799 & -0.6902 & -0.7089 & -0.7342 & -0.7477 \\
3 & -4.6420 & 4.6420 & -0.6755 & -0.6851 & -0.7047 & -0.7286 & -0.7468 \\
4 & -4.6143 & 4.6143 & -0.6763 & -0.6857 & -0.7041 & -0.7290 & -0.7463 \\
\hline
\end{tabular}

\begin{tabular}{crrrrr}
\hline mesh & $\psi \mid \partial \Omega_{3}^{6}$ & $\psi \mid \partial \Omega_{3}^{7}$ & $\psi \mid \partial \Omega_{3}^{8}$ & $\psi \mid \partial \Omega_{3}^{9}$ & $\psi \mid \partial \Omega_{3}^{10}$ \\
\hline 1 & -0.7252 & -0.7120 & -0.6877 & -0.6655 & -0.6533 \\
2 & -0.7437 & -0.7351 & -0.7120 & -0.6968 & -0.6859 \\
3 & -0.7494 & -0.7379 & -0.7155 & -0.6933 & -0.6785 \\
4 & -0.7492 & -0.7381 & -0.7157 & -0.6939 & -0.6793 \\
\hline
\end{tabular}

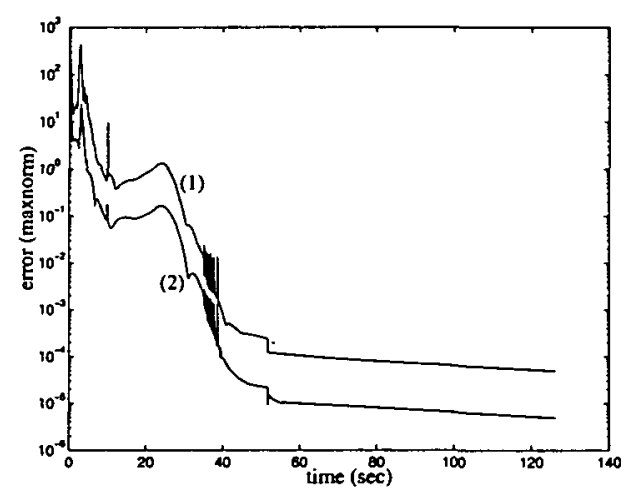

(a)

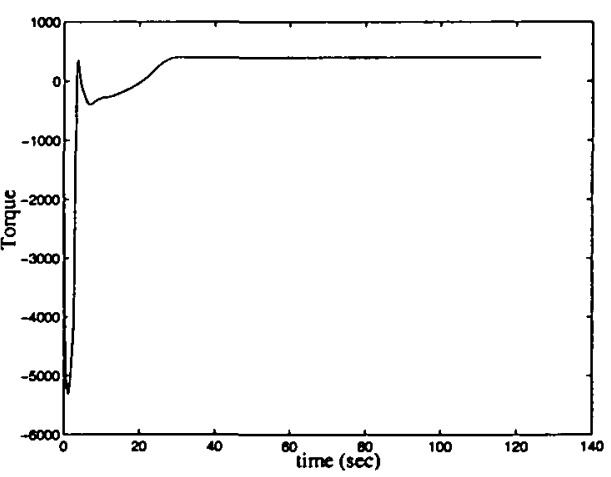

(b)

Figure 5. (a) Absolute errors for (1): $\left\|\tilde{Z}^{k+1}-\tilde{Z}^{k}\right\|_{\infty}$ and (2): $\left\|\tilde{\Psi}^{k+1}-\tilde{\Psi}^{k}\right\|_{\infty}$. (b) Torque along the rotor when $R e=1000, \bar{U}=10.0$ and $\bar{\epsilon}=0.5$.

As steady-state (converged) solutions are required, the long-time behaviour of the solution fields is examined. The discretised systems of equations are solved using a CGS scheme together with preconditioning. Therefore, a convergence criterion based 
on the difference in solution vectors at successive time steps is adequate. Figure 5 displays the time history of the maximum norm of the absolute error in both the stream and vorticity functions at successive time steps for the problem $\{1000.0,0.5,10.0\}$. Over the time taken to reach the steady-state solution, the error in successive vorticity iterates is always larger than that in the stream function iterates. This is true for all the problems solved in this paper and so a convergence criterion based on vorticity also ensures accuracy in the stream function. This has also been pointed out by Gupta and Manohar [12]. Furthermore, the torque exerted on the rotor in all the problems considered here reaches steady-state quickly.

6.1. Case 1: The bounding cylinders rotate in the same direction For the problems discussed in this section, the flow field is determined by computing the streamlines. However, we note that the contour levels shown are not uniform, this being done to highlight the main features without unnecessarily cluttering the flow field.

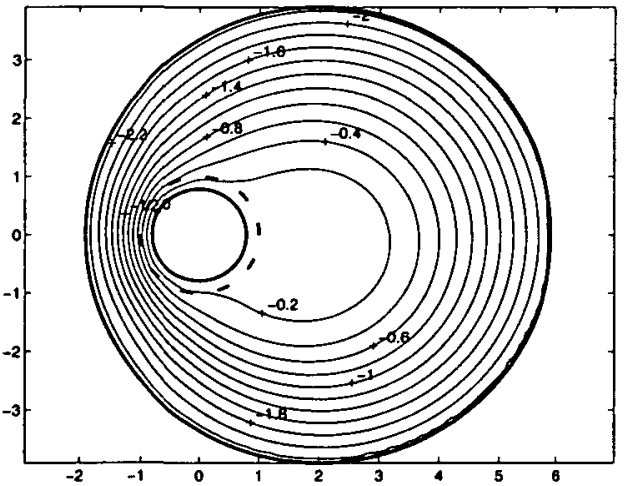

(a) $\bar{U}=1.7$

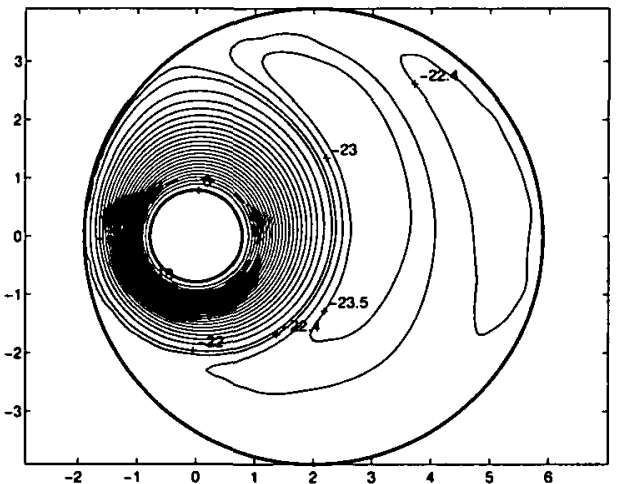

(b) $\bar{U}=2.0$

FIGURE 6. Contour plots for $R e=500, \bar{\epsilon}=0.7$, and various $\vec{U}$.

When the modified viscometer is placed within the bowl, increasing the rotational speed of the rotor can cause the generation of eddies within the flow domain. Figure 6 gives the contour plots when problems $\{500.0,0.7,1.7\}$ and $\{500.0,0.7,2.0\}$ are solved. For small $\bar{U}$ no eddies are present and the interaction of the fluid around the sleeve can clearly be seen. The amount of mixing depends on the eccentricity of the system. As $\bar{U}$ increases, a critical value is reached and a pair of eddies are formed within the flow domain. The critical value is dependent on the eccentricity. For example, when eccentricities of 0.5 and 0.7 are used, no eddies are observed when $\bar{U}$ is below 7.0 and 2.0 respectively. When eddies exist, the eddy closest to the sleeve (primary eddy) rotates clockwise and represents the dominant eddy within the system. The eddy located along the outer cylinder (secondary eddy) rotates in 


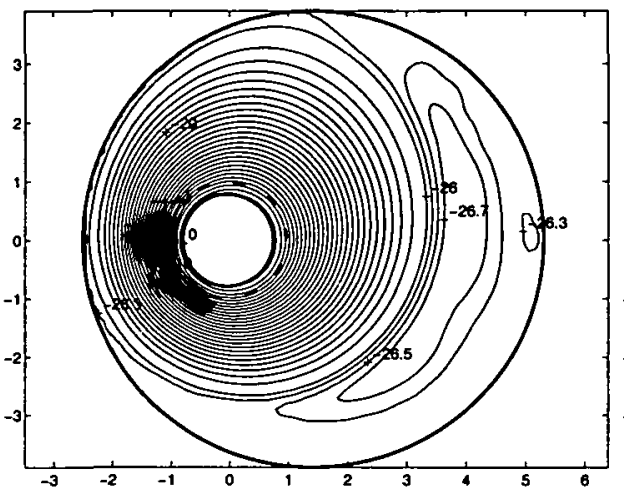

(a) $\bar{\epsilon}=0.5$

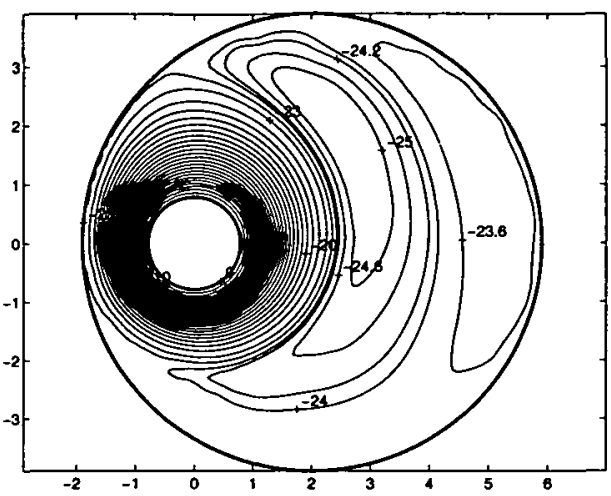

(b) $\bar{\epsilon}=0.7$

FIGURE 7. Contour plots for $\operatorname{Re}=500, \bar{U}=10.0$, and various $\bar{\epsilon}$.

a counter-clockwise direction. From Figure 6, the flow through the slotted sleeve is restricted when these eddies are present. Increasing the speed of the rotor does not affect, qualitatively, the flow field, or inhibit further the flow through the slotted sleeve; only the computed values for the stream function, vorticity and torque are affected. By solving problems $\{500.0,0.7,2.0\},\{500.0,0.7,5.0\}$, and $\{500.0,0.7,10.0\}$, Table 2 gives the torque exerted along the rotor $(\mathrm{T})$, the centre of the primary eddy, as well as the stream function $\left(\psi_{c}\right)$ and vorticity $\left(\zeta_{c}\right)$ there. Increasing $\bar{U}$ decreases both the torque exerted on the rotor and the stream function at the centre of the primary eddy, while the vorticity there increases.

TABLE 2. Values $\psi$ and $\zeta$ at $\left(x_{c}, y_{\mathrm{c}}\right)$ and torque along the rotor for $R e=500.0, \bar{\epsilon}=0.70$ and varying $\bar{U}$.

\begin{tabular}{rcccc}
\hline $\bar{U}$ & $\left(x_{c}, y_{c}\right)$ & $\psi_{c}$ & $\zeta_{c}$ & $T$ \\
\hline 2.0 & $(2.20,2.29)$ & -24.21 & -7.63 & 1470.85 \\
5.0 & $(2.20,2.29)$ & -24.58 & -7.50 & 1216.37 \\
10.0 & $(2.20,2.29)$ & -25.49 & -7.07 & 858.48 \\
\hline
\end{tabular}

TABLE 3. Values of $\psi$ and $\zeta$ at $\left(x_{c}, y_{c}\right)$, and torque along the rotor for $\bar{\epsilon}=0.5, \bar{U}=10.0$, and varying Reynolds number.

\begin{tabular}{rcccc}
\hline$R e$ & $\left(x_{c}, y_{c}\right)$ & $\psi_{c}$ & $\zeta_{c}$ & $T$ \\
\hline 50.0 & $(3.93,0.00)$ & -23.66 & -3.81 & 256.32 \\
100.0 & $(3.93,0.00)$ & -25.94 & -3.80 & 354.21 \\
500.0 & $(3.93,0.00)$ & -26.92 & -3.64 & 396.45 \\
1000.0 & $(3.93,0.00)$ & -27.00 & -3.62 & 399.72 \\
\hline
\end{tabular}




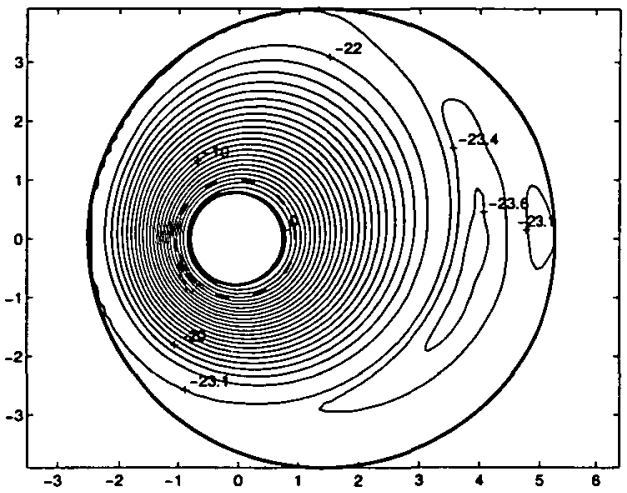

(a) $\operatorname{Re}=50.0$

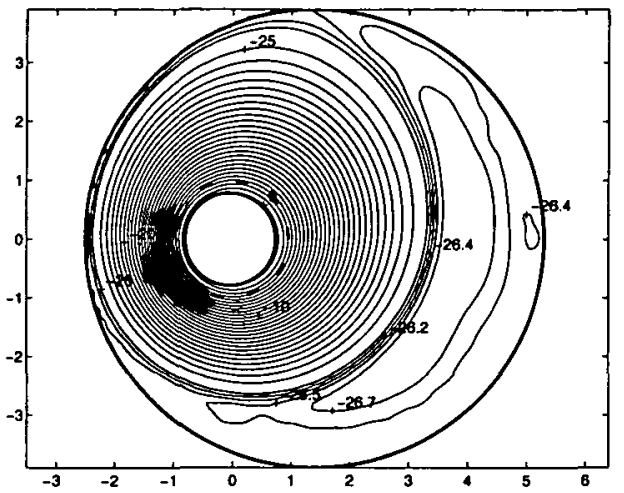

(b) $R e=1000.0$

FIGURE 8. Contour plots for $\bar{\epsilon}=0.5, \bar{U}=10.0$, and various Reynolds numbers.

Varying the eccentricity of the system influences both the presence of eddies and their location. Consider problems $\{500.0,0.5,10.0\}$ and $\{500.0,0.7,10.0\}$; their solution fields are given in Figure 7. For small eccentricities, and sufficiently large $\bar{U}$, the centrifugal force produced by the rotor enables circular flow to extend far into the main body of the domain. As the eccentricity is increased, the effect of this inertial force is reduced and the eddies produced cover a larger area of fluid. Furthermore, the centre of the primary eddy moves in the direction of the rotor. This is confirmed from the results given in the third line of Tables 2 and 3 which also show that both $\psi_{c}$ and the torque $T$ increase while $\zeta_{c}$ decreases with increasing eccentricity.

To study the effect of Reynolds number on the flow field, problems $\{50.0,0.5,10.0\}$, $\{100.0,0.5,10.0\},\{500.0,0.5,10.0\}$, and $\{1000.0,0.5,10.0\}$ are solved. Figure 8 plots the stream function obtained for the extreme values of the Reynolds number. These results, together with the contour plot given in Figure 7 for the case $\operatorname{Re}=500.0$, suggest that the position of the primary eddy is unaffected by the change in Reynolds number. This is verified in Table 3 which also shows that $\zeta_{c}$ and the torque $T$ increase while $\psi_{c}$ decreases as the Reynolds number is increased. Although, on physical grounds, the secondary eddy must exist, there is insufficient detail from the results given to discuss its behaviour.

6.1.1. The vorticity distribution We expect the vorticity distribution to be influenced by the slotted sleeve, which, together with the rotor and the outer bowl, are sources of vorticity. At high Reynolds number, the vorticity should decay quickly away from these surfaces. To illustrate this, we consider the problem $\{1000.0,0.5,10.0\}$ and measure the vorticity values along two lines, one inclined at $45^{\circ}$ to the horizontal line joining the centres of the rotor and the outer bowl, and the other inclined at $50^{\circ}$ (see Figure 9(a)). The direction of traverse along the lines is from left to right in each 


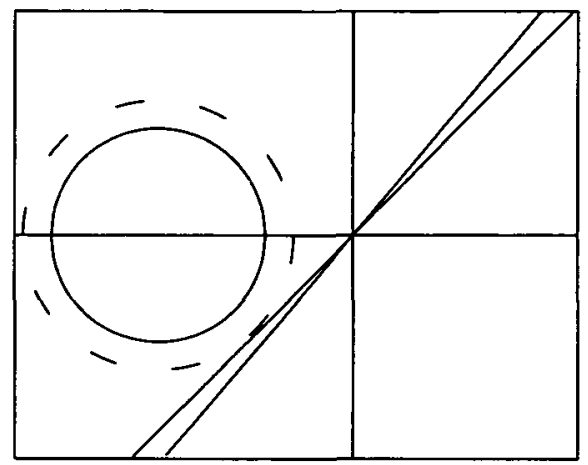

(a) The lines of traverse near the sleeve

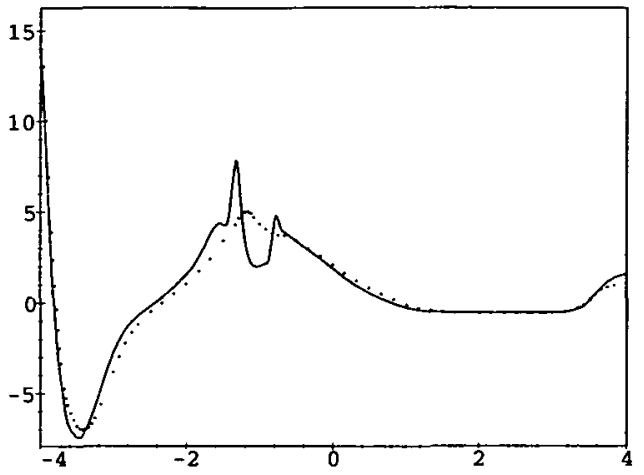

(b) $-45^{\circ}, \cdots 50^{\circ}$

FIGURE 9. Vorticity along two lines of traverse.

case. The $45^{\circ}$ line passes very close to the edges of two of the slots on the sleeve. The vorticity values along this line is shown by the solid curve in Figure 9(b). The two sharp peaks in the vorticity values away from the bowl along this curve are located close to the first and second slots below the horizontal line; the higher peak being near the edge closest to the line of traverse. The influence of this edge is still evident in the plot of vorticity values along the $50^{\circ}$ line of traverse, where a point plot is used to differentiate it from the previous traverse. The second peak along the $45^{\circ}$ line has all but disappeared along the $50^{\circ}$ line as the influence of the edges of the slots becomes weaker. Figure 9(b) also shows how the vorticity is convected away from the sleeve, with the swirl of the motion playing a part, as evidenced by the location of the peak values as one moves away from the sleeve.

The high Reynolds number flow between two eccentric rotating cylinders possesses a viscous boundary layer along each boundary. Away from the boundary layers the flow field consists of closed streamlines and, hence, the vorticity must be nearly constant in this region [2]. We anticipate that an inviscid core will only be present in the limited case when there is no flow through the slotted sleeve (that is, as $\bar{\epsilon} \rightarrow 0$ ), as the sleeve is a source of vorticity, and for problems $(\bar{\epsilon} \neq 0)$ when the prescribed tangential velocities do not produce a stagnation point along either bounding cylinders.

\subsection{Case 2: The bounding cylinders rotate in opposite directions For each} problem considered, a pair of contour plots is used to examine the flow field. The first contour plot is used to illustrate the entire flow field using a contour spacing of 0.2 , and the second contour plot describes the flow around the sleeve in more detail using a streamline spacing of 0.1 .

Consider the problems $\{500.0,0.2,-5.0\},\{500.0,0.5,-5.0\}$, and $\{500.0,0.7,-5.0\}$ whose solution fields are given in Figure 10. An eddy, rotating counter clockwise, is 
generated for an eccentricity as small as $\mathbf{0 . 2}$. From Figure 10, the presence of the eddy does not restrict the flow through the slotted sleeve, which is predominantly through the side closest to the eddy. As the eccentricity is increased, the eddy moves in the direction of rotation of the outer cylinder. This is verified from Table 4 which gives the torque, $T$, the eddy centre, as well as the stream function $\left(\psi_{c}\right)$ and vorticity $\left(\zeta_{c}\right)$ values there. In addition to the movement of the eddy, the flow through the sleeve increases with increasing eccentricity. According to Table 4, the torque exerted on the rotor and the vorticity at the eddy centre decrease, while the stream function at the eddy centre increases with increasing eccentricity.

TABLE 4. Values of $\psi$ and $\zeta$ at $\left(x_{c}, y_{c}\right)$, and torque along the rotor using meshes $\mathrm{A}, \mathrm{B}$, and $\mathrm{C}$.

\begin{tabular}{rcccc}
\hline $\bar{\epsilon}$ & $\left(x_{c}, y_{c}\right)$ & $\psi_{c}$ & $\zeta_{c}$ & $T$ \\
\hline 0.2 & $(1.27,-0.87)$ & 0.75 & 1.20 & 82.10 \\
0.5 & $(2.25,-0.36)$ & 1.24 & 0.80 & 76.04 \\
0.7 & $(2.81,-0.21)$ & 1.43 & 0.67 & 71.61 \\
\hline
\end{tabular}

The effect of the rotational speed of the rotor is studied by considering the problems $\{500.0,0.7,-5.0\},\{500.0,0.7,-10.0\}$, and $\{500.0,0.7,-20.0\}$. From Figure 11 we see that as the rotational speed of the rotor is increased (that is, $|\bar{U}|$ is increased), the eddy centre moves in the direction of the rotation of the rotor and the flow through the slots is restricted. Table 5 confirms the motion of the eddy and also shows that $\psi_{c}, \zeta_{c}$, as well as the torque, $T$, increase with increasing rotational speed.

TABLE 5. Values of $\psi$ and $\zeta$ at $\left(x_{c}, y_{c}\right)$, and torque along the rotor for $\operatorname{Re}=500.0, \bar{\epsilon}=0.7$, and varying $\bar{U}$.

\begin{tabular}{rcccr}
\hline $\bar{U}$ & $\left(x_{c}, y_{c}\right)$ & $\psi_{c}$ & $\zeta_{c}$ & $T$ \\
\hline-5.0 & $(2.81,-0.21)$ & 1.43 & 0.67 & 71.61 \\
-10.0 & $(2.85,-0.50)$ & 2.28 & 0.71 & 146.10 \\
-20.0 & $(2.91,-0.94)$ & 5.09 & 0.99 & 225.09 \\
\hline
\end{tabular}

Problems $\{50.0,0.5,-10.0\},\{100.0,0.5,-10.0\},\{500.0,0.5,-10.0\}$, and $\{1000.0$, $0.5,-10.0\}$ are solved in order to study the effect of the Reynolds number on the flow field. The contour plots for the extreme values of the Reynolds number are displayed in Figure 12 which indicates that the Reynolds number has little effect on the flow field. Table 6 shows that although the eddy centre is approximately independent of the Reynolds number, the computed values of the stream function and vorticity are affected by the parameter.

6.2.1. The vorticity distribution Comparing Figure 8(b) with Figure 12(b), we note that, apart from the eddy in Figure 12, the stream function contours are more 

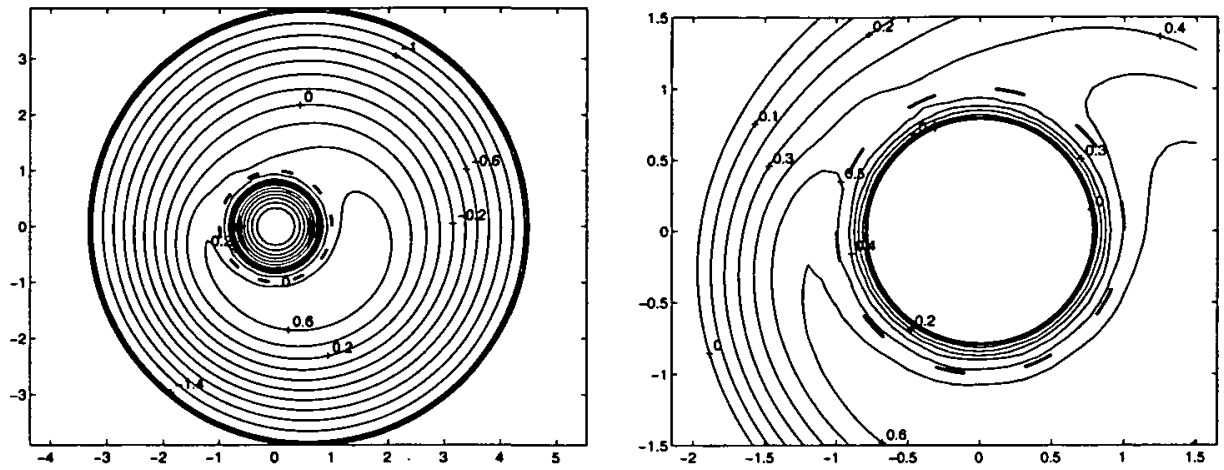

(a) $\bar{\epsilon}=0.2$
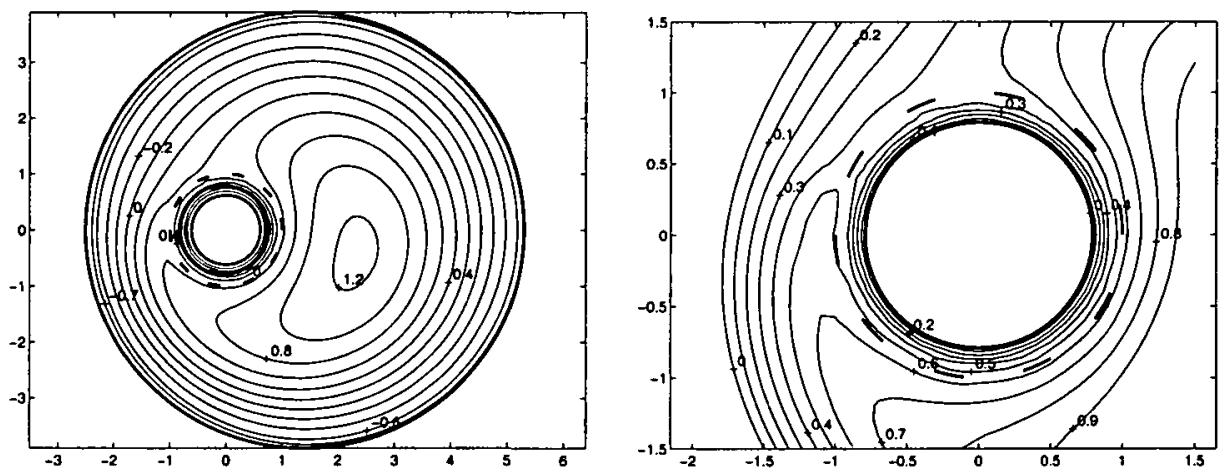

(b) $\bar{\epsilon}=0.5$
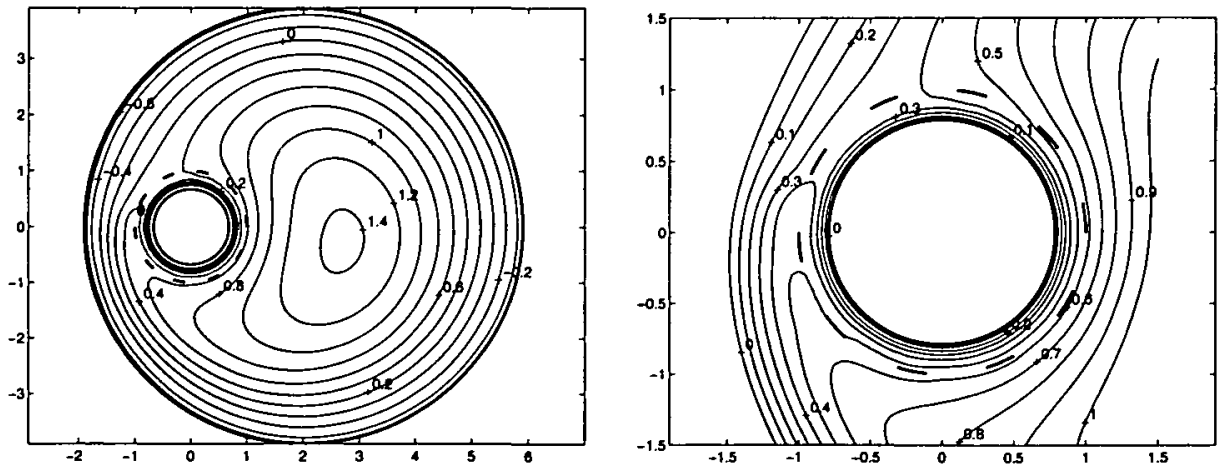

(c) $\bar{\epsilon}=0.7$

FIGURE 10. Contour plots in the entire flow field and around the sleeve for $\operatorname{Re}=500, \bar{U}=-5.0$, and various $\bar{\epsilon}$. 

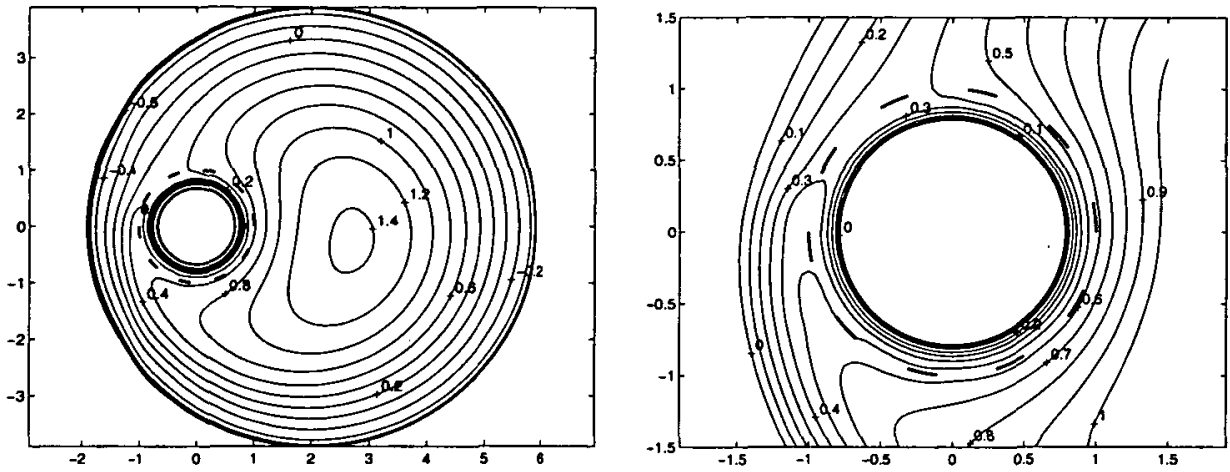

(a) $\bar{U}=-5.0$
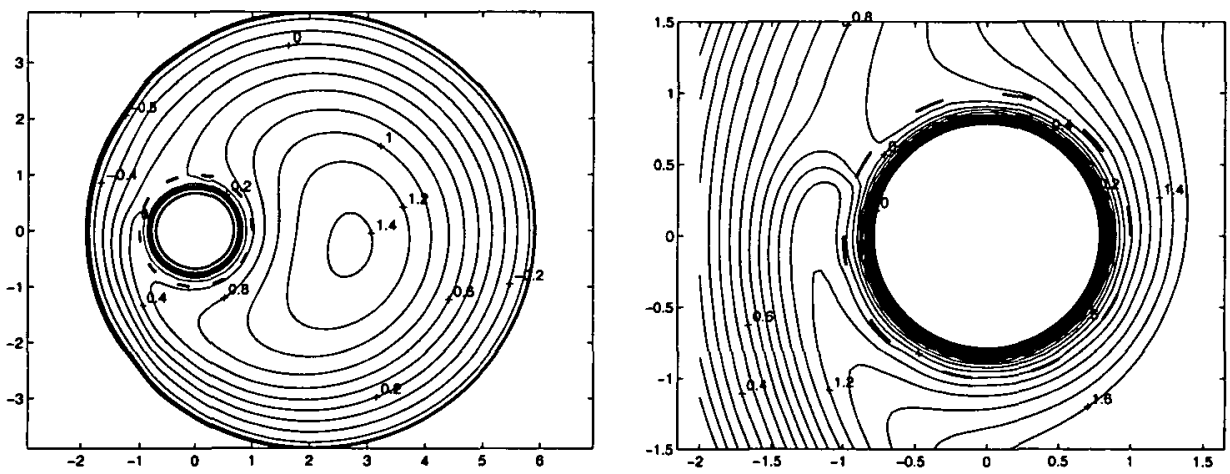

(b) $\bar{U}=-10.0$
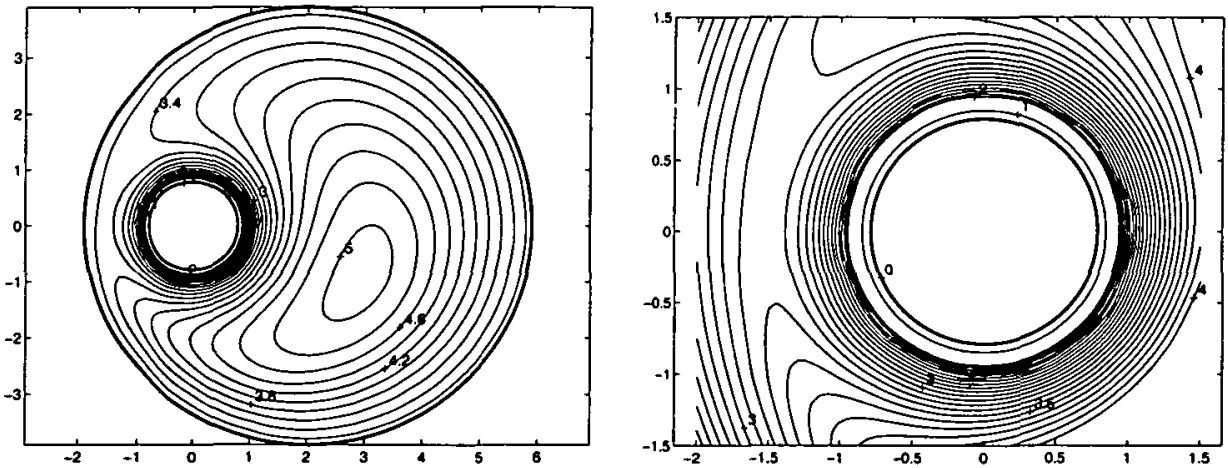

(c) $\bar{U}=-20.0$

FIGURE 11. Contour plots in the entire flow field and around the sleeve for $R e=500, \bar{\epsilon}=0.7$, and various $\bar{U}$. 

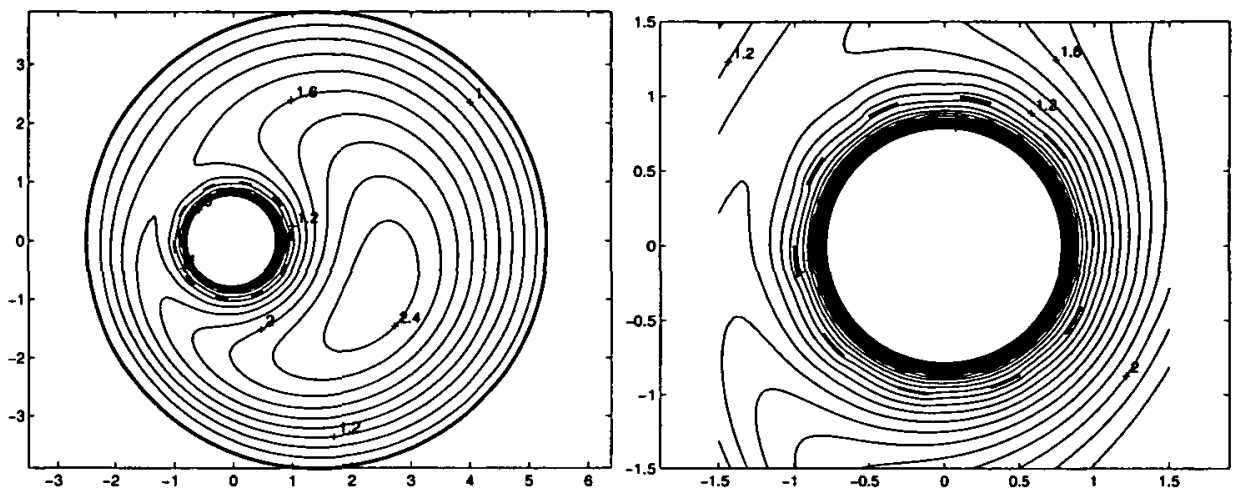

(a) $R e=50.0$
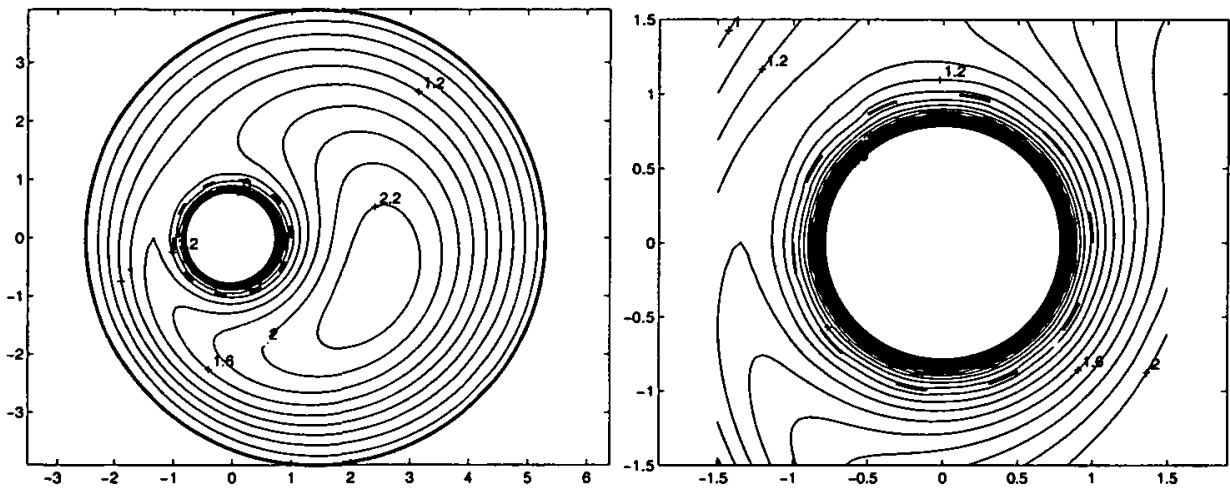

(b) $R e=1000.0$

FIGURE 12. Contour plots in the entire flow field and around the slotted sleeve for $\bar{\epsilon}=0.5, \bar{U}=-10.0$, and various Reynolds numbers.

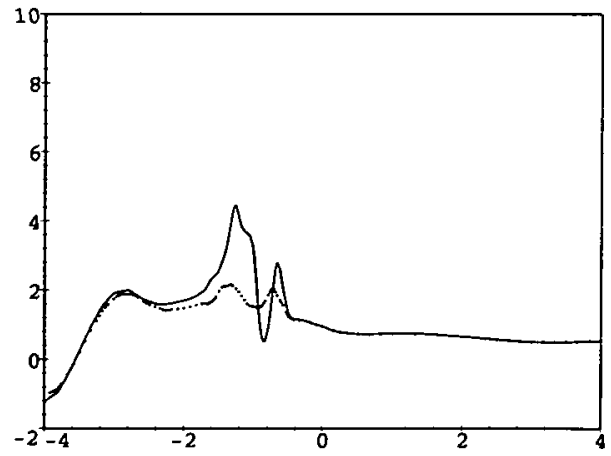

FIGURE 13. Vorticity along two lines of traverse $\left(-45^{\circ}, \ldots 50^{\circ}\right)$ 
TABLE 6. Values of $\psi$ and $\zeta$ at $\left(x_{c}, y_{c}\right)$, and torque along the rotor for $\bar{\epsilon}=0.5, \bar{U}=-10.0$, and varying Reynolds number.

\begin{tabular}{rcccc}
\hline$R e$ & $\left(x_{c}, y_{c}\right)$ & $\psi_{c}$ & $\zeta_{c}$ & $T$ \\
\hline 50.0 & $(2.39,-0.71)$ & 2.52 & 0.96 & 153.14 \\
100.0 & $(2.39,-0.71)$ & 2.47 & 0.95 & 137.98 \\
500.0 & $(2.39,-0.71)$ & 2.37 & 0.91 & 124.93 \\
1000.0 & $(2.39,-0.71)$ & 2.35 & 0.90 & 124.13 \\
\hline
\end{tabular}

evenly spaced in the counter-rotating case, except for the regions close to the slots of the sleeve. We expect that the generation of vorticity will be concentrated in the small neighbourhood about the sleeve. This is illustrated in Figure 13, which has been produced using a relatively coarse mesh, and, hence, has only qualitative relevance. The same two lines of traverse (see Figure 9(a)) are used, and the influence of the edges of the slots closest to the lines of traverse can be seen.

\section{Conclusion}

The flow between two eccentric rotating cylinders with a slotted sleeve placed around the inner cylinder was determined numerically using the exponentially fitted finite-volume method of Miller and Wang [18]. The flow field was determined for various Reynolds numbers, eccentricities, and rotational rates, when the cylinders rotate in the same sense (case 1) and in opposite senses (case 2).

For case 1, the flow field was characterised, for sufficiently large eccentricity and rotational rate, by the presence of two counter-rotating eddies within the main flow domain. The dominant eddy rotates clockwise while the subdominant eddy located along the outer cylinder rotates counter clockwise. The presence of these eddies restricted the flow through the slotted sleeve. Increasing the rotational speed of the inner cylinder had little effect on the flow field; however, as the eccentricity increased, the eddies moved in the direction of rotation of the bounding cylinders.

For case 2, it was shown that eddies occur within the flow field for eccentricities as small as 0.2 . As the eccentricity increased the eddy was seen to move in the direction of the rotation of the outer cylinder and the presence of the eddy promoted the flow through the slotted sleeve. When the rotational speed of the inner cylinder increased, the eddy moved in the direction of the inner cylinder.

No inviscid core was observed for either case 1 or case 2 for the high Reynolds number flow. The presence of the slotted sleeve allowed the vorticity generated there to be convected into the main flow domain.

Finally, we note that for the high Reynolds number flow between two eccentric rotating cylinders, Chipman and Duck [5] showed that the existence of a stagnation 
point on the bounding cylinders is dependent on the relative rotational rate of the cylinders and the eccentricity of the system. For example, from their Figure $3 b, a$ stagnation point along the boundary layer on the inner cylinder occurs when $\bar{U} \approx 5.0$ and $\bar{\epsilon} \approx 0.35$, and a stagnation point located on the outer cylinder is found when $|\bar{U}| \approx 1.0$ and $\bar{\epsilon} \approx 0.35$. No Reynolds numbers are given at these instances as the existence of a critical Reynolds number above which the flow in the boundary layers fails to be unidirectional is currently an open problem. Furthermore, according to Rosenhead [21, pages 505-506], this stagnation-point boundary-layer flow can also cause centrifugal instability. The connection between Reynolds number and centrifugal instability has been demonstrated experimentally. For example, in the experimental work of Verteegen and Jankowski [24] conditions for the generation of Taylor vortices are given. From their Figure 7 instability first occurs when, using our parameters, $\operatorname{Re} \approx 300.0$ and $\bar{U} \approx 2.0$ for an eccentricity of 0.5 . Although no direct comparison can be made with the results given in [24] or [5], the problems considered here are well beyond the conditions stated above, albeit using a relatively coarse mesh, we suspect the presence of the slotted sleeve may have a stabilising effect. The stability of the model considered here needs to be investigated in order to understand fully the role of the slotted sleeve on the flow field.

\section{References}

[1] A. J. Baker, "Finite element solution algorithm for viscous incompressible fluid dynamics", Intl. J. Num. Meth. Eng. 6 (1973) 89-101.

[2] G. K. Batchelor, "On steady laminar flow with closed streamlines at large Reynolds number", $J$. Fluid. Mech. 1 (1956) 177-190.

[3] A. Brandt and I. Yavneh, "Inadequacy of first-order upwind difference schemes for some recirculating flows", J. Comput. Phys. 93 (1991) 128-143.

[4] A. Campion-Renson and M. J. Crochet, "On the stream function - vorticity finite element solutions of Navier-Stokes equations", Intl. J. Num. Meth. Eng. 12 (1978) 1809-1818.

[5] P. D. Chipman and P. M. Duck, "On the high-Reynolds-number flow between non-coaxial rotating cylinders", Q. J. Mech. Appl. Math. 46 (2) (1993) 163-191.

[6] G. Comini, M. Manzan and C. Nonino, "Finite element solution of the streamfunction-vorticity equations for the incompressible two-dimensional flows", Intl. J. Num. Meth. Fluids 19 (1994) 513-525.

[7] M. J. Crochet, A. R. Davies and K. Walters (eds.), Numerical simulation of non-Newtonian flow (Elsevier, Amsterdam, 1984).

[8] R. C. DiPrima and J. T. Stuart, "The nonlinear calculation of Taylor-vortex flow between eccentric rotating cylinders.", J. Fluid. Mech. 67 (1) (1975) 85-111.

[9] R. Glowinski and O. Pironneau, "Numerical methods for the first biharmonic equation and for the two-dimensional Stokes problem", SIAM Rev. 21 (2) (1979) 167-212.

[10] P. M. Gresho, "Some current CFD issues relevant to the incompressible Navier-Stokes equations", Comp. Meth. Appl. Mech. Eng. 87 (1991) 201-252. 
[11] M. D. Gunzburger and J. S. Peterson, "Finite-element methods for the streamfunction-vorticity equations:boundary-condition treatments and multiply connected domains.", SIAM J. Sci. Stat. Comput. 9 (4) (1988) 650-668.

[12] M. M. Gupta and R. P. Manohar, "Boundary approximations and accuracy in viscous flow computations", J. Comput. Phys. 31 (1979) 265-288.

[13] L. D. Hird and P. F. Siew, "Small Reynolds number flow between eccentric rotating cylinders with a permeable sleeve", J. Austral. Math. Soc. (Ser. B) 38 (2) (1996) 255-273.

[14] M. Hriberšek and L. Škerget, "Iterative methods in solving Navier-Stokes equations by the boundary element method", Intl. J. Num. Meth. Eng. 39 (1996) 115-139.

[15] M. Ikegawa, "A new finite element technique for the analysis of steady viscous flow problems", Intl. J. Num. Meth. Eng. 14 (1979) 103-113.

[16] The MathWorks Inc., MATLAB:high-performance numeric computation and visualization software, 1995.

[17] J. A. Meijerink and H. A. van der Vorst, "An iterative solution method for linear systems of which the coefficient matrix is a symmetric M-matrix", Math. Comp. 31 (137) (1977) 148-162.

[18] J. J. H. Miller and S. Wang, "An exponentially fitted finite volume method for the numerical solution to 2D unsteady incompressible flow problems", J. Comput. Phys. 115 (1) (1994) 56-64.

[19] J. J. H. Miller and S. Wang, "A new non-conforming Petrov-Galerkin finite-element method with triangular elements for a singularly perturbed advection-diffusion problem", IMA J. Num. Anal. 14 (1994) 257-276.

[20] I. J. Overend, R. R. Horsley, R. L. Jones and R. K. Vinycomb, "A new method for the measurement of rheological properties of settling slurries", in IXth Intl. Congress. Rheol., (1984), 583-590.

[21] L. Rosenhead (ed.), Laminar boundary layers (Dover, New York, 1963).

[22] P. Sonneveld, "CGS, a fast lanczos-type solver for nonsymmetric linear systems", SIAM J. Sci. Stat. Comput. 10 (1) (1989) 36-52.

[23] C. Taylor and P. Hood, "A numerical solution for the Navier-Stokes equations using the finite element technique", Comp. Fluids 1 (1973) 73-100.

[24] P. L. Versteegen and D. F. Jankowski, "Experiments on the stability of viscous flow between eccentric rotating cylinders", Phys. Fluids 12 (6) (1969) 1138-1143.

[25] W. W. Wood, "The asymptotic expansions at large Reynolds numbers for steady motion between non-coaxial rotating cylinders", J. Fluid. Mech. 13 (2) (1957) 159-175.

[26] L. B. Zhang, "A second-order upwinding finite difference scheme for the steady Navier-Stokes equations in primitive variables in a cavity with a multigrid solver.", RAIRO Modél. Math. Anal. Numér. 24 (1) (1990) 133-150. 